\title{
Impact of Adaptive Loading in Scheduling Algorithms for OFDMA Systems
}

\author{
Ricardo B. dos Santos*, Francisco Rafael M. Lima*, Walter C. Freitas Jr. and Francisco Rodrigo P. Cavalcanti
}

\begin{abstract}
In this article we evaluate the impact of optimal Adaptive Loading in conventional scheduling algorithms in Orthogonal Frequency Division Multiple Access networks. The gain in performance by the utilization of Adaptive Loading over Adaptive Modulation with equal power allocation are different according to the scheduling algorithm.
\end{abstract}

Keywords-OFDMA, round robin, rate maximization, rate adaptive, adaptive loading, adaptive modulation and radio resource algorithms.

Resumo-Neste artigo é avaliado o impacto de algoritmos de alocação de potência e bit em redes OFDMA (do inglês, Orthogonal Frequency Division Multiple Access) utilizando algoritmos de escalonamento convencionais. $O$ ganho em desempenho devido ao uso de algoritmos de alocação de potência e bit sobre os esquemas de modulação adaptativa com distribuição igualitária de potência são diferentes de acordo com o algoritmo de escalonamento empregado.

Palavras-Chave-OFDMA, round robin, maximização de taxa, adaptação de taxa, adaptação de potência e bit, modulação adaptativa e algoritmos de alocação de recursos de rádio.

\section{INTRODUCTION}

Fourth Generation (4G) and future wireless mobile communications systems, such as Long Term Evolution (LTE) of 3rd. Generation Partnership Project (3GPP), have adopted Orthogonal Frequency Division Multiple Access (OFDMA) as the multiple radio access technology. Some advantages of OFDMA schemes compared to the others are the robustness against the effects of multipath, the higher spectral efficiency caused by the orthogonality of subcarriers, and the flexibility to scale these schemes to larger bandwidths. Besides these advantages, OFDMA offers many possibilities to Radio Resource Allocation (RRA) improve the system performance. In OFDMA based systems the subcarriers, bit and power can be dynamically allocated to each User Equipment (UE) by exploiting the frequency and multi-user diversities.

The dynamic allocation of power and modulation among subcarriers, called Adaptive Loading (AL), has been the focus of several articles in the literature [1], [2], [3], [4]. These works have evaluated the impact of this technique in point-to-point connections. Some of the conclusions are that $\mathrm{AL}$ schemes provide interesting gains compared to static schemes with fixed power and modulation. However, the difference in performance of AL and Adaptive Modulation (AM), where

\footnotetext{
* These students are supported by a scholarship granted by FUNCAP. This work was supported by a grant from Ericsson of Brazil - research branch under ERBB/UFC.18 technical cooperation contract. The authors are from Wireless Telecommunication Research Group - GTEL / Department of Teleinformatics - DETI / Federal University of Ceará - UFC. Emails: \{brauner,rafaelm,walter,rodrigo\}@gtel.ufc.br
}

the power is fixed among subcarriers and only the modulation is adapted, is not large depending on the channel model.

Another work [5] has aimed at evaluating the impact of the adaptation of subcarrier and power allocation over static schemes in point-to-multipoint scenario. It showed that full dynamic schemes with subcarrier allocation and $\mathrm{AL}$ provide huge gains over static schemes. The authors also compared the performance of dynamic subcarrier allocation and power allocation separately. However, the number of dynamic resource allocation algorithms evaluated in this work was limited.

The gains obtained by using $\mathrm{AL}$ algorithms in the multi-user scenario depends on some factors. Some of them are the amount of subcarriers assigned to each UE and the channel state of assigned subcarriers. Note that these characteristics are strictly correlated with which scheduling algorithm is configured in the system. Scheduling algorithms can have different objectives and therefore they manage the system resources differently.

In this paper, we evaluate the performance of different scheduling algorithms with $\mathrm{AL}$ and $\mathrm{AM}$. The remainder of the paper is organized as follows. Section II presents the system modeling. Section III shows the description of the evaluated scheduling algorithms and the AL algorithm utilized in this work. Finally in sections IV and V, the numerical results achieved by simulation and the conclusions are provided, respectively.

\section{System Modeling}

In the modeled OFDMA system the subcarriers are modeled as $N$ flat Rayleigh fading channels correlated in time and frequency. The subcarriers are shared by $J$ terminals using a full data buffer service. In addition, a fluid model is assumed for the traffic, i.e., the UE data is completely divisible. The assignment of subcarriers to the UEs is represented by $\mathbf{X}[k]$, that is composed by the elements $x_{j, n}[k]$ which assumes the value 1 when the subcarrier $n$ is assigned to UE $j$ at Transmission Time Interval (TTI) $k$, and 0 otherwise.

The Base Station (BS), that has a total power constraint of $p_{\max }$, is assumed to have knowledge of the channel gain $g_{j, n}[k]$ of each UE $j$ in each subcarrier $n$ at TTI $k$. The Signal-to-Noise Ratio (SNR) $\gamma_{j, n}[k]$ of UE $j$ in subcarrier $n$ at TTI $k$ is defined as

$$
\gamma_{j, n}[k]=\frac{g_{j, n}[k] \cdot p_{n}[k]}{\sigma^{2}}
$$

where $p_{n}[k]$ is the transmit power in subcarrier $n$ at TTI $k$ composing the power vector $\mathbf{p}[k]$, and $\sigma^{2}$ is the Additive White Gaussian Noise (AWGN) noise power. 


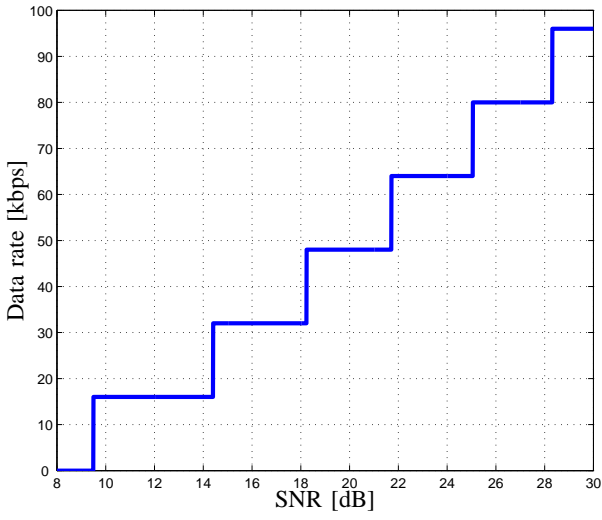

Fig. 1. Link adaptation curve.

To represent the finite Modulation and Coding Schemes (MCSs) of the system, the capacity curve was sampled in the M-Quadrature Amplitude Modulation (QAM) modulations ( $M=2^{m} ; m=1,2,3,4,5$ and 6 ) as can be seen in Fig. 1 . The MCS available in the system is in set $\mathcal{D}$. The function that maps $\gamma_{j, n}[k]$ in $\mathcal{R}_{j, n}[k]$ will be represented by $F(\cdot)$ in the remaining of this work. Furthermore, the temporal index, $k$, will be omitted in the following for simplicity. Our link adaptation curve is shown in Fig. 1. This curve represents the data rate to be employed in a subcarrier depending on subcarrier instantaneous SNR.

The transmit data rate $\mathcal{R}_{j, n}[k]$ of UE $j$ in subcarrier $n$ at TTI $k$ is given by [6]

$$
\mathcal{R}_{j, n}[k]=w \cdot \log _{2}\left(1+\frac{\gamma_{j, n}[k]}{\Gamma}\right)
$$

where $w$ is the subcarrier bandwidth and

$$
\Gamma=-\frac{\ln (5 \cdot B E R)}{1.5}
$$

is the SNR gap for the system Bit Error Rate (BER) requirements. The allocated data rate to the UE $j$ at TTI $k$ is represented by $r_{j}[k]$.

Once the subcarrier and bit allocation are defined by the scheduler, it is assumed that this information are sent via a separate control channel.

\section{AlgORITHM DESCRIPTIONS}

In this section we present the description of the scheduling algorithms studied in this work, followed by the AL algorithm.

\section{A. Scheduling}

This section is devoted to the evaluated scheduling algorithms focused in this paper. The objectives and features of Rate Maximization (RM), Rate Adaptive (RA) and Round Robin (RR) schemes are presented in the following. Note that all scheduling schemes presented were modified to take decisions based on equal power allocation and provide only the subcarrier assignment. After that, in case of the evaluation of AL schemes, the power is adapted per subcarrier.
1) Rate Maximization: One of the first problems studied in OFDMA RRA was the RM. The objective of this algorithm is to maximize the sum of the UE data rates subject to the constraint that one subcarrier cannot be shared by more than one UE at the same time and the total BS power constraint. The problem formulation is presented in equation 4 .

$$
\begin{aligned}
\underset{\mathbf{p}, \mathbf{X}}{\max } & \sum_{j} \sum_{n} F\left(\frac{p_{n} \cdot g_{j, n}}{\sigma^{2}}\right) \cdot x_{j, n} \\
\text { subject to } & \\
& \sum_{j} x_{j, n} \leqslant 1, \quad \text { for all } n \\
& \sum_{n} p_{n} \leqslant p_{\max } .
\end{aligned}
$$

The algorithm utilized to solve this problem is presented in Algorithm 1. In this algorithm, each subcarrier has to be assigned to the UE that has the highest channel gain on it. After that, an AL algorithm is utilized to allocate power and bits among subcarriers.

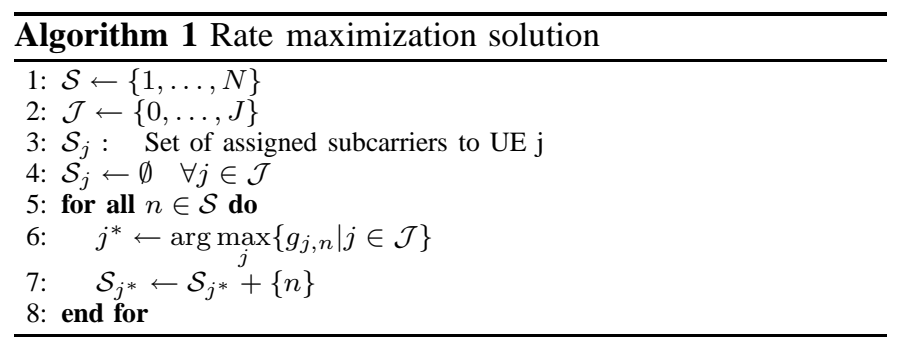

In spite of its simple solution, the RM problem is not suitable when one considers fairness issues. This algorithm can cause starvation of UEs at cell edge due to high path loss [7].

2) Rate Adaptive: In the RA approach, the objective is to maximize the lowest UE data rate, subject to the constraint that one subcarrier cannot be shared by more than one UE at the same time and the total BS power constraint. The problem formulation is presented in equation 5 .

$$
\begin{aligned}
& \underset{\mathbf{p}, \mathbf{X}}{\max } \in \\
& \text { subject to } \\
& \sum_{j} x_{j, n} \leq 1 \text { for all } n \\
& \quad \sum_{n} p_{n} \leq p_{\max } \\
& \quad \sum_{n} F\left(\frac{p_{n} \cdot g_{j, n}}{\sigma^{2}}\right) \cdot x_{j, n} \geq \epsilon \text { for all } j .
\end{aligned}
$$

The RA problem belongs to the group of combinatorial programming ones that have no immediate solution. Rhee et al. proposed a sub-optimal solution to the RA problem in [8] and concluded that its performance is near to the optimal one. The Rhee's solution is in Algorithm 2.

In Algorithm 2, it is assumed that the power is equally distributed among subcarriers to ease the subcarrier assignment 


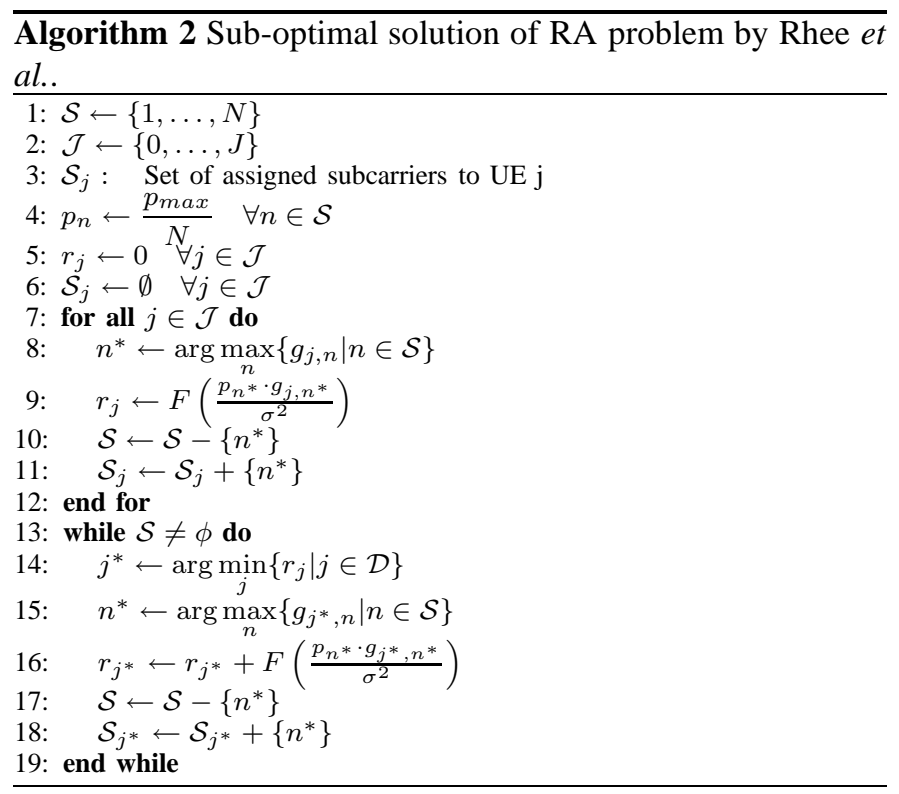

(see step 3). The algorithm is composed by two loops. The first one allocates the best subcarrier of each UE to assure fairness (see step 6). After that, the subcarriers are assigned sequentially to the UE that has the lowest achieved data rate (see step 11). Note that, the subcarrier assigned in the second loop is also the one in best channel state among the remaining. By the intrinsic characteristics of the RA problem, this strategy tends to balance the data rates achieved by the UE.

3) Round Robin: The RR algorithm has the purpose of allocating the same time share to all UE in the system [9]. In an OFDMA system, this is performed by allocating transmission opportunity at each TTI in a cyclic way to the UE in the system. Therefore, in a given TTI, all subcarriers and transmit power available in the system are allocated to only one UE. Another relevant point regarding RR scheduling algorithm, is that it does not utilize any channel state information.

\section{B. Adaptive Loading}

In this article, we utilize an optimal AL algorithm patented by Hughes-Hartogs [10]. The algorithm is presented in Algorithm 3.

In our studies, the Algorithm 3 is performed in each UE individually, i.e., it is run after subcarrier assignment done by the algorithms explained in section III-A. The total available power assumed for each UE is proportional to the number of subcarrier assigned to it.

In the algorithm initialization, it is necessary to calculate the amount of power necessary to achieve MCS $m$ in subcarrier $n$, pow $w_{m, n}$, as in step 3 . To work out this, it is used the channel gain information in each subcarrier that was assigned to a given UE and the link adaptation curve shown in Fig. 1. Then, $\Delta$ pow $_{m, n}$, that is the power necessary to increase from the MCS level $m-1$ to the $m$ in subcarrier $n$ is calculated (see step 4). Note that this power can be different according to the channel gain and modulation level. The main idea of Algorithm 3 is very simple: allocate bits, i.e., increase the MCS level to the subcarriers that requires the lower amount of

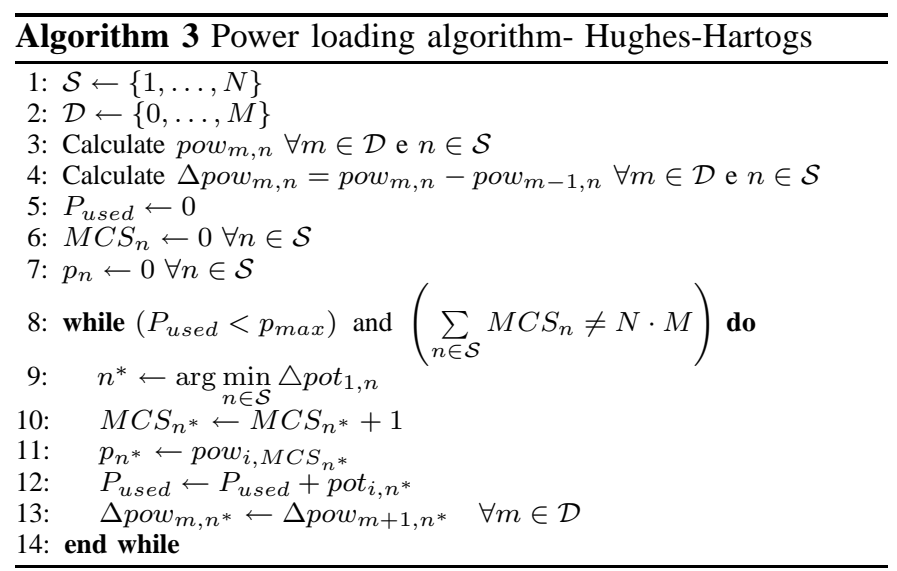

power to achieve this according to the link adaptation (see step 9). This is performed until all subcarriers reach the maximum MCS level or the UE available power is completely utilized (see step 8). In the end, this algorithm achieves the maximum data rate achieved with the power constraint and subcarriers channel states provided.

Despite of optimality, this algorithm is time expensive regarding computation processing. As an example, considering that there are $N$ subcarriers and $M$ available modulation levels, this algorithm would perform $M \cdot N$ loops in the worst case. Furthermore, it is necessary to calculate the matrix pow $_{m, n}$ and $\Delta$ pow $_{m, n}$, that have dimensions $M \times N$.

\section{RESUlts}

In this section we present the numerical results obtained from computational simulations. In this paper we study the case of a single cell system. The main parameters used in the simulations are shown in the following.

The central operating frequency is $2 \mathrm{GHz}$. It is considered that there are 150 subcarriers available in the cell with a bandwidth of $15 \mathrm{kHz}$ for each subcarrier. Regarding propagation, the path loss $L[\mathrm{~dB}]$ at distance $d[\mathrm{~km}]$ is calculated by $L=128.1+37.6 \cdot \log _{10}(d)$. The shadowing standard deviation is $8 \mathrm{~dB}$ and the fast fading model is Typical Urban (TU).

The modeled power budget has the following values. The noise power is $-123.24 \mathrm{dBm}$ per subcarrier, the total base station power is $20 \mathrm{~W}$, and the cell radius is $1 \mathrm{~km}$. Also, we assumed that the UEs are static and uniformly distributed in the cell coverage area. The minimum distance between the UEs and the cell antenna is $10 \mathrm{~m}$. The resource allocation takes place in a TTI of $0.5 \mathrm{~ms}$.

For each load presented, 200 realizations were performed in order to achieve statistical confidence. Moreover, the realizations have the time length of 300 TTIs. This time length was sufficient to achieve convergence of average UE data rates for all simulated algorithms.

We simulated loads ranging from 6 to 39 UEs for two scenarios: equal power with $\mathrm{AM}$ and $\mathrm{AL}$. We present results of average cell data rate for all simulated loads for both scenarios. Furthermore, we present the Cummulative Distribution Function (CDF) of the UE average data rate in 


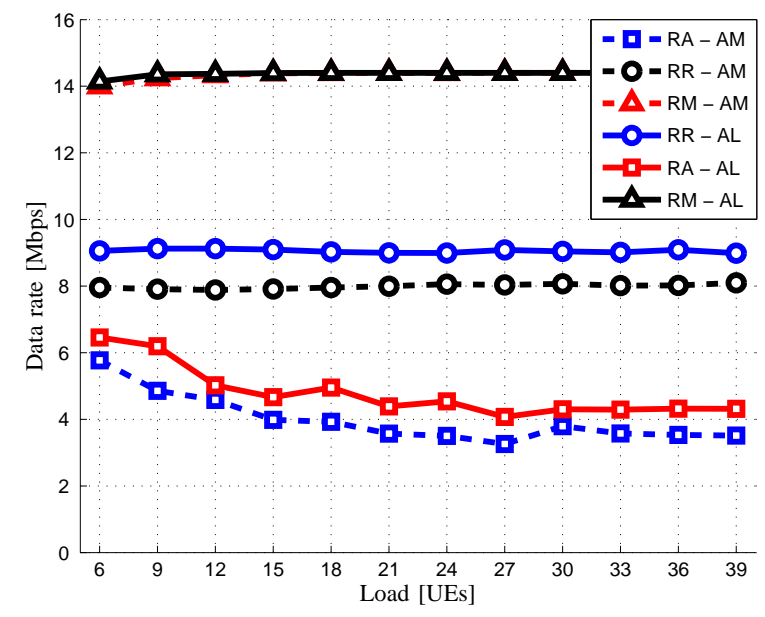

Fig. 2. Average total cell data rate of loads from 6 to 39 UEs.

all realizations for a low and a high load. The performance of each of the simulated algorithms is presented in data rate.

Fig. 2 shows results of average total cell data rate for each algorithm and simulated load. Focusing firstly on the RM algorithm, we can see that, as it was expected, the RM algorithm outperforms the other algorithms in the total cell data rate in both scenarios. This can be explained by the fact that this algorithm exploits the multi-user and frequency diversities, in such a way, that it schedules only the UEs with the best channel conditions in each subcarrier. Also note that the total cell data rate has a light increase with the system load due to the higher multi-user diversity.

As the RM algorithm already schedules only the UEs with the best channel conditions, only a small data rate improvement can be provided by the AL algorithm. So in the Fig. 2 the performance of the RM with AL and with only AM are almost identical.

The RR algorithm has an intermediate performance regarding total cell data rate when compared with the other algorithms. Its performance do not varies with the load and this behavior can be explained by the algorithm approach. As all UEs have opportunity to transmit using the entire bandwidth sequentially, the average total cell data rate is the average of the data rate achieved by all UEs in their transmission opportunity. This makes the total cell data rate depend only on the channel state of the UEs in the cell resulting in the same performance for all loads.

With respect to the AL performance with the RR scheduler, it has the greatest increase in performance of all presented algorithms. This can be explained by the fact that, as only one UE is scheduled at each TTI, the single-user AL algorithm has a higher degree of freedom to optimize the use of the power in each subcarrier. This power efficiency reflects in the UE data rates.

The RA problem has the objective of balancing the data rate of all UEs. So, as the data rate of all UEs will be nearly the same, the total cell data rate depends only on the data rate that can be achieved by all UEs at the same time. The results on Fig. 2 show that the average cell data rate decreases

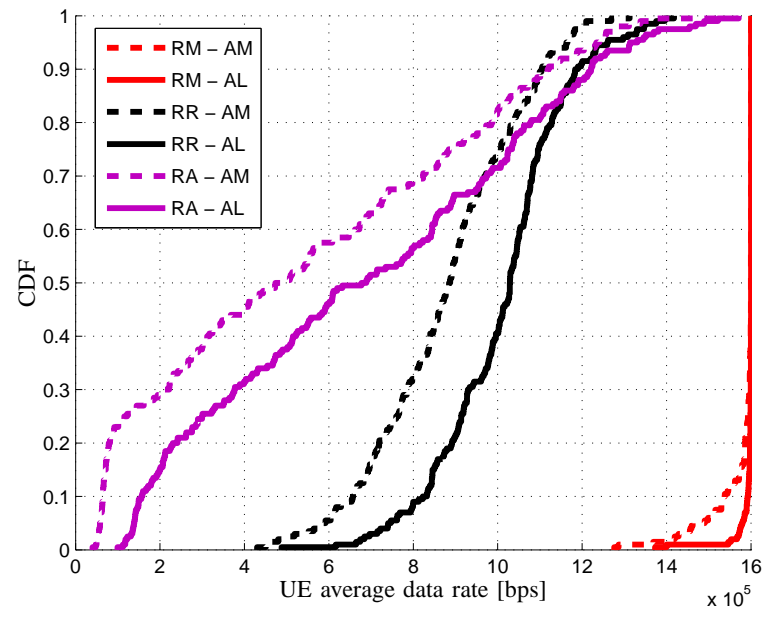

Fig. 3. CDF of the average UE data rate for the 9 UEs load.

with the increase of the load. This happens because when load increases, the absolute number of UEs with poor channel condition increases too. As the number of resources necessary to compensate for each UE with poor channel is very high, the data rate achieved by the UEs decreases with the increasing of the number of poor UEs, resulting in the increase of the load. As can be seen in Fig. 2, the RA had a clear performance gain in total cell data rate with the AL algorithm.

The Fig. 3 presents the CDF of the UE average data rate for simulated realizations with the RM, RR and RA algorithms, with AM and AL for the 9 UEs load. The UE average data rate for the RM are almost the same on $75 \%$ of the realizations and the average data rate is exactly the channel maximum total data rate divided by the number of UEs in the system. This happens because as the subcarriers are allocated to the UEs in best channel conditions, the equal power allocation already provides the highest modulation level to the subcarriers. In Fig. 3 is also shown that the AL gives real gains only on $25 \%$ of the realizations. Those realizations represent the rare cases in which the UE with the best channel condition in a given subcarrier does not achieve the maximum data rate on it using equal power. In this case the AL was capable to optimize the use of the power and reach data rates close to the maximum capacity.

The results in Fig. 3 enforce those on the Fig. 2 showing an intermediary performance for the RR solution providing data rates higher than those provided by the RA in $85-90 \%$ of the realizations. The RR data rate performance is worse than the RM one and this happens because RR provides fairness with respect to the number of resources provided to each UE and this fairness comes with a decrease of the spectral efficiency. We can also see that, for the RR, the AL provides a good increase in the data rate performance and the reason for this gain, as explained before, is that the whole bandwidth is allocated to one UE at time.

As stated before, the objective of balancing the data rate of all UEs makes the RA very dependent on the channel condition of the UEs on the cell. One UE with a very poor channel is 


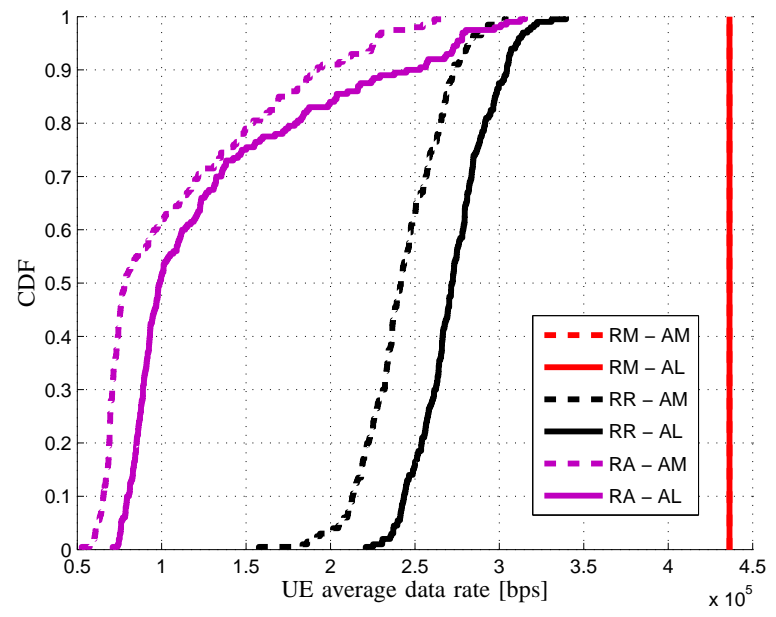

Fig. 4. CDF of the average UE data rate for the 33 UEs load.

sufficient to strongly degrade the cell data rate performance. This explain why the RA has the worst performance in $85-90 \%$ of the realizations. However, in realizations that only have UEs with good channel conditions (the other 10-15\%), the average data rate is greatly increased beating the RR data rate. The great impact that the channel of a UE can have on the system performance explains why very different UE average data rates on the presented realizations resulting in a range of UEs average data rate much broader than the range of the other algorithms. It is also important to notice that the AL provides an acceptable gains in all realizations. As in such low loads the number of subcarriers allocated to each UEs should be still comparable the those achieved in the RR algorithm.

In Fig. 4 we present the CDF of the UE average data rate for the realizations simulated for the RM, RR and RA algorithms, with AM and AL for the 33 UEs load. The average data rates are lower than the ones in Fig. 3 because now the system resources have to be shared among more UEs, resulting in a lower achieved data rate. For the RM algorithm, the highest MCS was achieved in all subcarriers for all realizations resulting in the maximum data rate for all realizations. The cases where some subcarriers do not achieve the maximum data rate as in the $33 \mathrm{UE}$ load, do not exist anymore due to higher multi-user diversity in this load. As the highest possible cell data rate is already achieved, the AL can not give any performance gain.

Even with different absolute values, the results for the RR in Fig. 4 have a similar behavior to those shown in Fig. 3 and the AL still provide considerable gains in data rate performance.
The results for the RA algorithm present a higher occurrence of the lower data rates than in Fig. 3. As explained before, this is a result of the higher number of UEs with poor channel condition. The AL performance, however, is lower than the one on Fig. 3 because, as more UE share the resources, less subcarriers are allocated to each UE. Therefore, less subcarriers result in lower gains with AL.

\section{CONCLUSION}

In this work we presented some Orthogonal Frequency Division Multiple Access Radio Resource Allocation solutions in the literature and analyzed the impact of an optimal single-user Adaptive Loading on their performance. We concluded that the Adaptive Loading can provide higher performance gains when large numbers of subcarriers are allocated for the same User Equipment like in the Round Robin solution. We also concluded that solutions that allocate subcarriers in very good state to the User Equipments like the Rate Maximization, have little or none performance gain. And finally, concluded that solutions like the Rate Adaptive that still allocate subcariers to User Equipments with poor channel condition but do not allocated large numbers of subcarriers to one User Equipment have acceptable performance gains with the use of Adaptive Loading.

\section{REFERENCES}

[1] T. Willink e P. Wittke, "Optimization and Performance Evaluation of Multicarrier Transmission", IEEE Trans. on Information Theory, v. 43, n. 2, p. 426-440, 1997.

[2] A. Czylwik, "OFDM and Related Methods For Broadband Mobile Radio Channels", Proc. of Broadband Communications, p. 91-98, 1998.

[3] H. Rohling e R. Gruenheid, "Performance of an OFDM-TDMA Mobile Communication System”, Proc. of IEEE VTC, v. 3, p. 1589-1593, May 1996.

[4] A. Czylwik, "Adaptive OFDM for Wideband Radio Channels", Proc. of the Global Telecommunications Conference, v. 2, p. 713-718, 1996.

[5] M. Bohge, "Bit Loading Versus Dynamic Subcarrier Assignment in Multi-User OFDM-FDMA Systems", Diploma Thesis at Technical University of Berlin, Germany, September 2004.

[6] I.C. Wong, Zukang Shen, B.L. Evans and J.G. Andrews, "A Low Complexity Algorithm for Proportional Resource Allocation in OFDMA Systems," IEEE Workshop on Signal Processing Systems, p.1-6, October 2004.

[7] J. Gross and M. Bohge, "Dynamic Mechanisms in OFDM Wireless Systems: A Survey on Mathematical and System Engineering Contributions", Tech. Rep. TKN-06-001, Telecommunication Networks Group, Technische Universitat Berlin, May 2006.

[8] W. Rhee and J. Ciof, "Increase in Capacity of Multiuser OFDM System Using Dynamic Subchannel Allocation", Proc. vehicular technology Conference (VTC), p. 1085-1089, 2000.

[9] F. Berggren and R. Jantti, "Asymptotically Fair Scheduling on Fading Channels,"Proc. of IEEE VTC'02 Fall, p. 1934-1938, September 2002.

[10] D. Hughes-Hartogs, "Ensemble Modem Structure for Imperfect Transmission Media”, U.S. Patents N. 4.679.227 (July 1987), 4.731.816 (March 1988) e 4.833.796 (May 1989). 